\title{
Score Evaluation of MRI Sequence Type Related Artifacts after Interbody Fusion with Metallic Implants-A Spine Specimen Study
}

\author{
Thorsten Ernstberger $^{1 *}$, Gottfried Buchhorn², Gabert Heidrich ${ }^{3}$ \\ ${ }^{1}$ Clinic for Spinal Surgery, Klinikum Bad Bramstedt, Bad Bramstedt, Germany \\ ${ }^{2}$ Biomaterial Laboratory, Department of Orthopaedic Surgery, University of Göttingen, Göttingen, Germany \\ ${ }^{3}$ Center of Radiology Weilheim, Ambulatory Healthcare Center, Weilheim i. Obb, Germany \\ Email: *t.ernstberger@klinkumbb.de
}

Received February 1, 2012; revised March 16, 2012; accepted April 20, 2012

\begin{abstract}
Introduction: According to anterior spine fusion intervertebral disc spacers made of titanium or cobalt-chromium alloys are of special interest. With regard to postoperative problems implant related artifacts can lead to a decreased MRI evaluation. The focus of this study was to compare the respective implant artifact range dependend on different MRI sequences. To simplify artifact evaluation we introduced in this study a new developed 0-1-2 score. Material and Methods: We performed an MRI artifact evaluation of 2 different metallic intervertebral disc spacers (cobalt-chromiumand titanium alloy). A carcass porcine spine was employed. Considering 12 defined spinal regions of interest we evaluated the respective implant artifact properties independent from the total artifact volume by using a new developed 0-1-2 score. The artifact range was documented for 15 different MRI-sequences. Results: For the titanium spacer as well as the cobalt-chromium-spacer an MRI evaluation of the implant/disc space situation could not be carried out. In contrast to the cobalt chromium spacer the titanium spacer allowed a good differentiation of the spinal canal opposite to the implant. Optimal MRI imaging results for both metallic intervertebral disc spacers could be achieved considering TSE sequences. Conclusion: A comparison of these two metallic spacers showed in all examined sequences clear advantages in favour of the titanium spacer. The best MRI representation of both tested implants by reducing implant related artifacts could be achieved with fast spin echo (TSE) sequences. In spite of the use of TSE sequences a variability of susceptibility artifacts has to be included with regard to implant shape and material. With regard to the results of this study the easy use of a new developed artifact score represented a useful help to compare implant related MRI artifact properties independent from the actual implant related total artifact volume.
\end{abstract}

Keywords: Metallic Intervertebral Disc Spacer; Artifact Score; MRI; Susceptibility Artifacts

\section{Introduction}

Regarding to spine diseases the magnetic resonance imaging is considered as a standard procedure for preoperative diagnostic. Especially disc pathologies as well as changes of the spinal canal can be represented with high precision.

According to anterior spine fusion different intervertebral disc spacers are of special interest. The implant design is dependent on the respective material as well as implantation characteristics. Considering postoperative complications after lumbar vertebra fusion magnetic resonance imaging can be necessary. In this context implant related artifacts can lead to a decreased evaluation of the MRI scan. Recent studies have shown that artifact disturbances particularly with metallic implants can be

"Corresponding author. minimized by modifications of routine MRI sequences [1]. On account of such experiences the present cadaveric study should demonstrate, to what extent implant related MRI artifacts can affect an evaluation of intervertebral disc spacers under special consideration to neighbouring structures. For easy evaluation of implant related artefact dimensions, we conducted a new score, which was developed on the basis of defined anatomical regions of interest. In this context the susceptibility artifacts of 2 different metallic intervertebral disc spacers was documented for 15 MRI sequences and possible implant related factors were discussed.

\section{Material and Methods}

In this study we performed a magnetic resonance imaging evaluation of 2 implanted metallic intervertebral disc 
spacers which differed in form, material, surface qualities and implantation characteristics (Figures 1(a) and (b)). We employed the spine of a slaughted pig. After preparation the paravertebral muscles including the surrounding skin as well as the psoas muscles were kept. Special attention was applied to the maintenance of the neurological structures of the spinal canal. Anatomically the pig myelon reaches up to the vertebra $\mathrm{S} 2$, therefore, were considered the distal third of the thoracic spine as well as the whole lumbar spine. To estimate the maximally possible artifact extension, we carried out a first MRI evaluation with a cobalt-chromium intervertebral disc spacer considering its ferromagnetic material properties. This spacer was completely surrounded with a homogeneous coat of meat. Regarding to different MRI-sequences a maximum artifact width of $6.5 \mathrm{~cm}$ could be calculated. Considering this result we determined the implantation distances of both metallic disc spacers.

\subsection{Implants}

Intervertebral disc dowel (IDD: ESKA Implants GmbH \& Co, Lübeck, Germany, Figures 1(a) and (b-A).

Material: Cobalt-chromium-molybdenum.

This metallic implant demonstrates a cylindric, slightly conical bullet shape. The implant length was $35 \mathrm{~mm}$, the diameter amounted to $15 \mathrm{~mm}$. On the one side this implant demonstrates a rounded cap. The implant surface has a patended metallic structure. This surface configure-
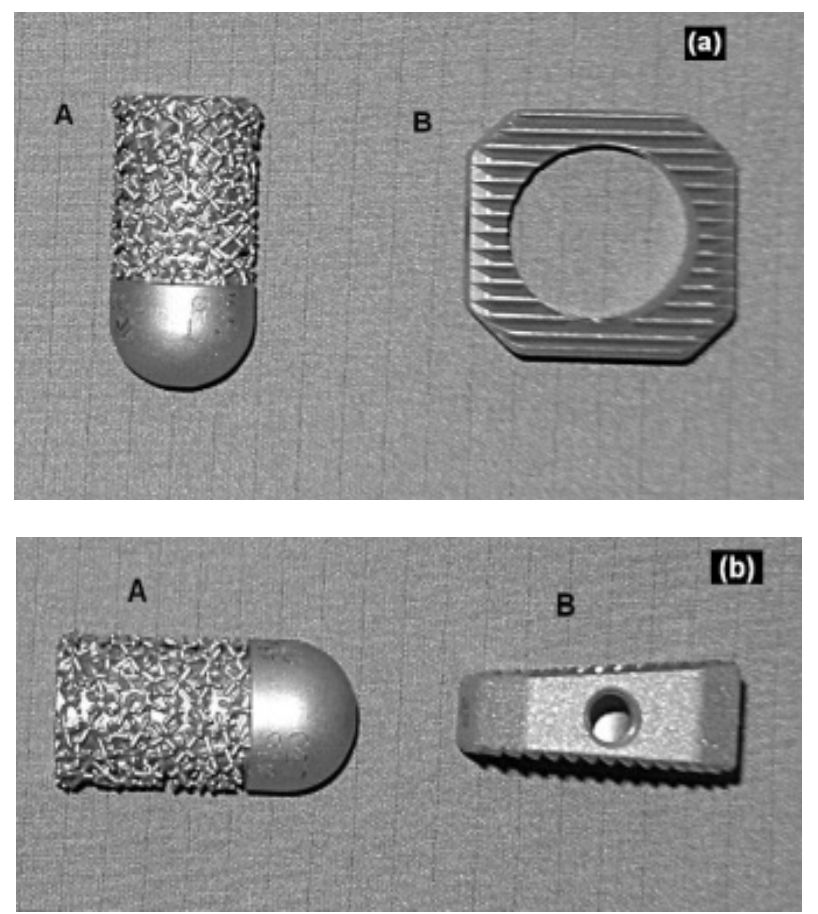

Figure 1. Implants. A: Intervertebral disc dowel (Cobaltchromium-molybdenum) on top + lateral view; B: IBS-spacer (Titanium-aluminium-vanadium) on top + lateral view. tion shows similarities to spongy bone.

Intervertebral Body Spacer (IBS LIBERTY: Peter Brehm Company, Weisendorf, Germany: Figures 1(a) and (b-B).

Material: Titanium-aluminium-vanadium

Considering an implant height of $10 \mathrm{~mm}$ the IBS spacer has a square shape of $25 \times 25 \mathrm{~mm}$ with a dorsal slope of 7 degrees of the upper surface in anteroposterior direction.

The implant surfaces demonstrates a tooth ribs configuration. In addition, this implant represents a central drilling of $15 \mathrm{~mm}$ for a possible spongiosa accomodation.

\subsection{Implantation}

In accordance to human the spinal column of the porcine specimen shows a vertebral size increase in craniocaudal direction. In this connection the lower lumbar vertebrae represents a maximum size of $25 \times 25 \times 20 \mathrm{~mm}$ (height $\times$ width $\times$ depth).

The examined implants showed an oversizing in contrast to the respective intervertebral disc space. In this context we included an implant extension into the spinal canal. The preparation of the lumbar and thoracic disc spaces were performed considering a possible central positioning of the examined implants. The paravertebral muscles including the skin as well as the psoas muscles were left. After intervertebral disc removing the respective intervertebral disc spacers were implanted. The maximally implantation depth was reached if the implant stand in line to the anterior vertebra front. Finally a conventional radiograph documentation was carried out (Figures 2(a) and (b)). After a first MRI test run an anterior supplementary meat coat was necessary for a sufficient referencing of the used implants.

\subsection{Magnetic Resonance Imaging}

All examinations were executed with a 1.5T-MRI (Magnetom-Symphony, Siemens AG. Medical Solution, Erlangen, Germany). Dependent on the respective MRIsequence we selected a matrix with $256 \times 256$, $384 \times 384$ or $512 \times 512$ combined with a Field of View (FOV) of 500 mms. With the exception of the T1 FLASH 2D sequence (slice thickness $5.5 \mathrm{~mm}$ ) and the T2 DESS 3D sequence (slice thickness $1.5 \mathrm{~mm}$ ) all other MRI sequences were performed with a slice thickness of $3 \mathrm{~mm}$. The examination record with all parameters are listed in Table 1. Beneath T1 spin echo (SE) and fast spin echo (TSE) sequences this protocoll also contained protone densitiy (PD) sequences as well as 2-D and 3-D sequences.

\subsection{Evaluation Unity}

A 0-1-2 scoring system was established to rank the MRI 
Table 1. MRI-sequence record.

\begin{tabular}{ccccccccc}
\hline Sequences & FA & TR & TE & ST & BW & FOV & Number of slices & Matrix \\
\hline T1 FLASH 2D & 70 & 181 & 4,8 & 5.5 & 260 & 500 & 19 & $256 \times 256$ \\
T1 FLASH 2D FS & 70 & 275 & 4,76 & 5.5 & 260 & 500 & 19 & $256 \times 256$ \\
T2 MEDIC 2D FS & 40 & 2660 & 27 & 3.0 & 70 & 500 & 40 & $256 \times 256$ \\
T1 FLASH 3D & 60 & 60 & 11 & 3.0 & 70 & 500 & 40 & $256 \times 256$ \\
T2 DESS 3D & 25 & 23.68 & 6.63 & 1.5 & 130 & 500 & 64 & $256 \times 256$ \\
TOF FISP 3D & 25 & 36 & 4.59 & 3.0 & 130 & 500 & 52 & $384 \times 384$ \\
T2 CISS 3D & 70 & 10.16 & 5.08 & 3.0 & 130 & 500 & 64 & $256 \times 256$ \\
T1 TSE & 150 & 2260 & 14 & 3.0 & 150 & 500 & 40 & $512 \times 512$ \\
T1 TSE var & 150 & 600 & 14 & 3.0 & 150 & 500 & 40 & $512 \times 512$ \\
T1 SE & 90 & 1270 & 14 & 3.0 & 90 & 500 & 40 & $512 \times 512$ \\
T1 SE var & 90 & 600 & 14 & 3.0 & 90 & 500 & 40 & $512 \times 512$ \\
T1 SE FS var & 90 & 684 & 14 & 3.0 & 90 & 500 & 40 & $512 \times 512$ \\
T2 TSE/PD & 150 & 6110 & 14 & 3.0 & 130 & 500 & 40 & $256 \times 256$ \\
T2 TSE/PD FS & 150 & 6760 & 14 & 3.0 & 130 & 500 & 40 & $256 \times 256$ \\
STIR & 180 & 10000 & 38 & 3.0 & 130 & 500 & 40 & $256 \times 256$ \\
\hline
\end{tabular}

FLASH = Fast Low Angle Shot; MEDIC = Multi Echo Data Image Combination; DESS = Dual Echoe Steady State; FS = Fat Saturated; FISP = Fast Imaging with Steady Precession; CISS = Constructive Interference in Stady State; SE = Spin Echo; TSE = Turbo Spin Echo; PD = Protone densitiy; STIR = Short Tau Inversion Recovery; TOF = Time of Flight; TR = Time of Repitition; TE = Time of Echo; FA = Flip Angle; ST = Slab Thickness; BW = Band Width; FOV = Field Of View; var = varied.
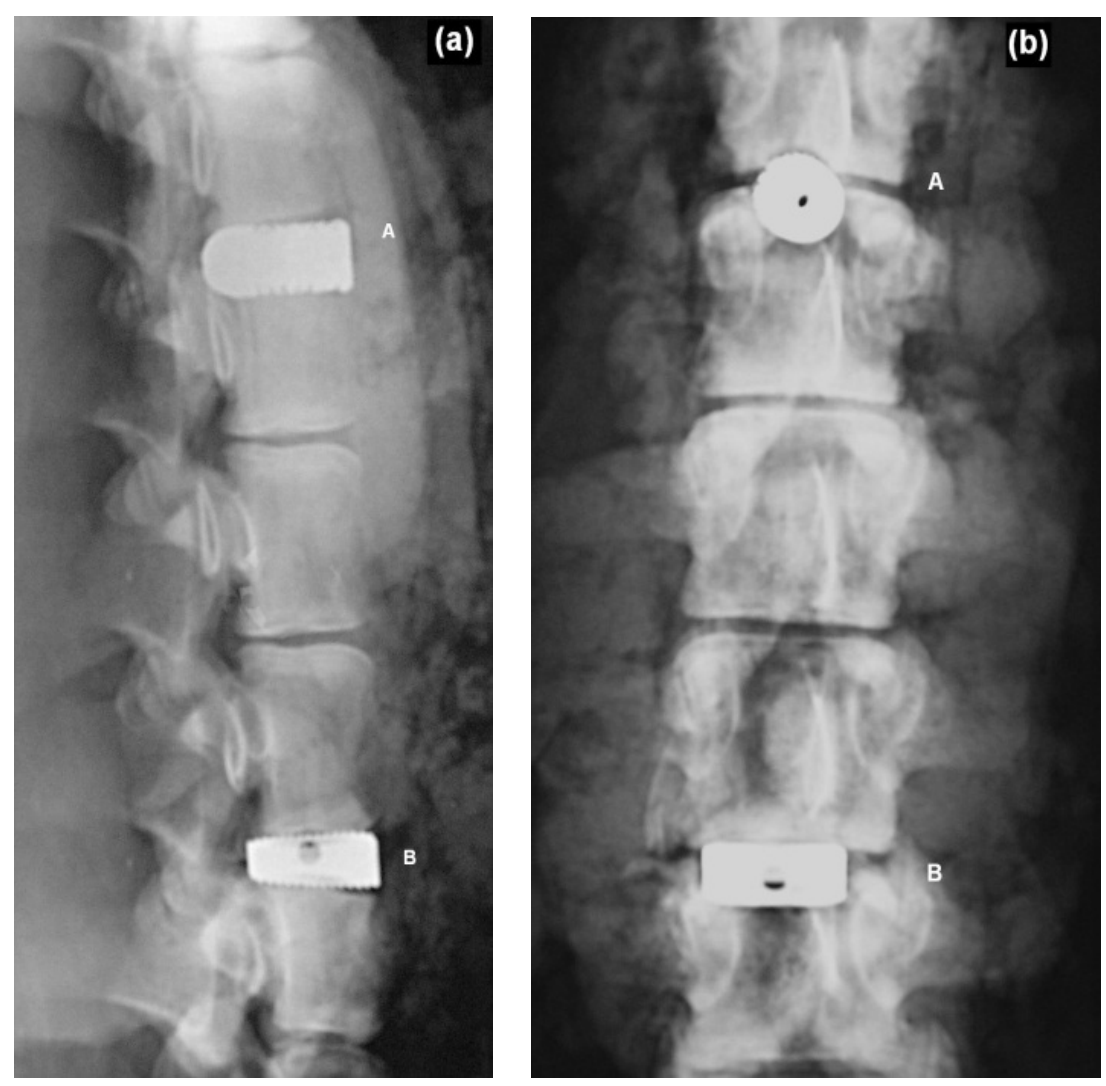

Figure 2. Conventional radiograph. A: Intervertebral disc dowel (lateral + coronal view); B: IBS-spacer (lateral + coronal view). 
scans. An evaluation unit was defined as 2 adjacent vertebrae encompassing the intervertebral disc space. 12 regions of interest (ROI) were demarcated (Figure 3). Every ROI could achieve a maximum score of 2 points. A total score of 24 points was equivalent to a score of $100 \%$. Two board-certified specialists (one radiologist (GH) and one spinal surgeon (TE)) experienced in reading spinal MRI evaluated the scans independently of each other. The evaluators scored regions as $0=$ not distinguishable, 1 = partly distinguishable and 2 = completely distinguishable. The interobserver validation of the scoring system across all 15 sequences was tested for statistical significance using a t test with a significance level of $\mathrm{P}=0.05$ (Tables 2 and 3).

\section{Results}

On the basis of 15 different MRI sequences an imaging scoring were performed considering implant related MRI artifacts (Figures 4(a)-(d)). A total score of maximally 24 points could be achieved. Per MRI sequence the achieved score for every region of interest were added. Dependent on the respective intervertebral disc spacers it should be demontstrated which MRI sequences guarantee an optimal implant imaging.

\subsection{Intervertebral Disc Dowel (IDD)}

According to the T1 TSE sequences (Figure 4(a-A)) the IDD could achieve a maximum total score of 17 points (Table 2). With regard to the relevant MRI scan the implant related susceptibility artifact could be clearly differentiated opposite the spinal surrounding. However, the determined regions of interest demonstrated a reduced MRI imaging quality of less than $50 \%$ (1 point). Only a limited evaluation of the implant-bone contact area was possible considering the artifact extension. Exclusively the distant vertebral end plates, anterior edge of the lower

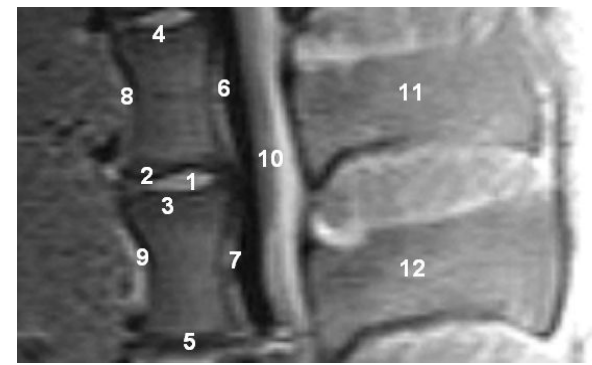

1. Intervertebral disc space; 2. Cranial adjacent plate; 3. Caudal adjacent plate; 4 . Cranial distant plate; 5 . Caudal distant plate; 6 . Posterior edge of the cranial vertebra; 7 . Posterior edge of the caudal vertebra; 8 . Anterior edge of the cranial vertebra; 9 . Anterior edge of the caudal vertebra; 10. Spinal canal; 11. Cranial spinous process; 12. Caudal spinous process.

Figure 3. MRI-evaluation unity with regions of interest (porcine carcass spine).
Table 2. Total score values considering different MRIsequences (1 = Spinal surgeon, 2 = Radiologist $)$.

\begin{tabular}{|c|c|c|c|c|}
\hline \multirow{2}{*}{ MRI Sequence } & \multicolumn{2}{|c|}{ IBS } & \multicolumn{2}{|c|}{ IDD } \\
\hline & 1 & 2 & 1 & 2 \\
\hline $\begin{array}{l}\text { T1 FLASH 2D } \\
\text { TR:181 TE:4 }\end{array}$ & 13 & 13 & 9 & 8 \\
\hline $\begin{array}{l}\text { T1 FLASH 2D FS } \\
\text { TR:275 TE:4 }\end{array}$ & 13 & 13 & 10 & 10 \\
\hline $\begin{array}{l}\text { T2 MEDIC 2D FS } \\
\text { TR:2660 TE:27 }\end{array}$ & 11 & 11 & 4 & 4 \\
\hline $\begin{array}{l}\text { T1 FLASH 3D FS } \\
\text { TR:60 TE:11 }\end{array}$ & 10 & 10 & 5 & 6 \\
\hline $\begin{array}{l}\text { T2 DESS 3D } \\
\text { TR:23 TE:6 }\end{array}$ & 13 & 12 & 6 & 6 \\
\hline $\begin{array}{l}\text { T2 CISS 3D } \\
\text { TR:10 TE:5 }\end{array}$ & 13 & 12 & 7 & 7 \\
\hline $\begin{array}{c}\text { T1 SE } \\
\text { TR:1270 TE:14 }\end{array}$ & 13 & 14 & 12 & 12 \\
\hline $\begin{array}{c}\text { T1 TSE } \\
\text { TR:2260 TE:14 }\end{array}$ & 15 & 15 & 12 & 12 \\
\hline $\begin{array}{c}\text { T1 TSE } \\
\text { TR:600 TE:14 }\end{array}$ & 15 & 15 & 12 & 12 \\
\hline $\begin{array}{c}\text { T1 SE } \\
\text { TR:600 TE:14 }\end{array}$ & 13 & 13 & 12 & 12 \\
\hline $\begin{array}{c}\text { T1 SE FS } \\
\text { TR:684 TE:14 }\end{array}$ & 13 & 13 & 8 & 7 \\
\hline $\begin{array}{c}\text { PD + T2 TSE } \\
\text { TR:6110 TE:14 }\end{array}$ & 13 & 13 & 8 & 8 \\
\hline $\begin{array}{c}\text { PD + T2 TSE FS } \\
\text { TR:6760 TE:14 }\end{array}$ & 13 & 13 & 7 & 7 \\
\hline $\begin{array}{c}\text { STIR } \\
\text { TR:10000 TE:38 }\end{array}$ & 13 & 13 & 10 & 10 \\
\hline $\begin{array}{l}\text { TOF FISP 3D } \\
\text { TR:36 TE: } 4\end{array}$ & 12 & 12 & 3 & 4 \\
\hline
\end{tabular}

Table 3. Interobserver validation across all 15 sequences.

\begin{tabular}{cccccc}
\hline & \multicolumn{2}{c}{ Mean score value } & \multicolumn{2}{c}{ Standard deviation } & \multirow{2}{*}{ P value } \\
\cline { 2 - 5 } & $\begin{array}{c}\text { Spinal } \\
\text { surgeon }\end{array}$ & Radiologist & $\begin{array}{c}\text { Spinal } \\
\text { surgeon }\end{array}$ & Radiologist & \\
\hline IBS & 12.87 & 12.8 & +1.25 & +1.32 & $\mathrm{P}=0.58$ \\
IDD & 8.33 & 8.33 & +3.02 & +2.85 & $\mathrm{P}=1.0$ \\
\hline
\end{tabular}

vertebra as well as the spinous processus (2 points respectively) demonstrated a sufficient MRI imaging. A clear differentiation of the spinal canal opposite the implant could not be performed ( 0 point). The artifact extension admitted neither conclusions on the implant position, nor on the implant surrounding. The lowest MRI imaging quality with a total score of 3 points could be demonstrated considering the TOF FISP 3D sequence (Figure 4(d-A)). In this context a usably implant differentiation opposite the spinal surrounding could be carried out in no way. 

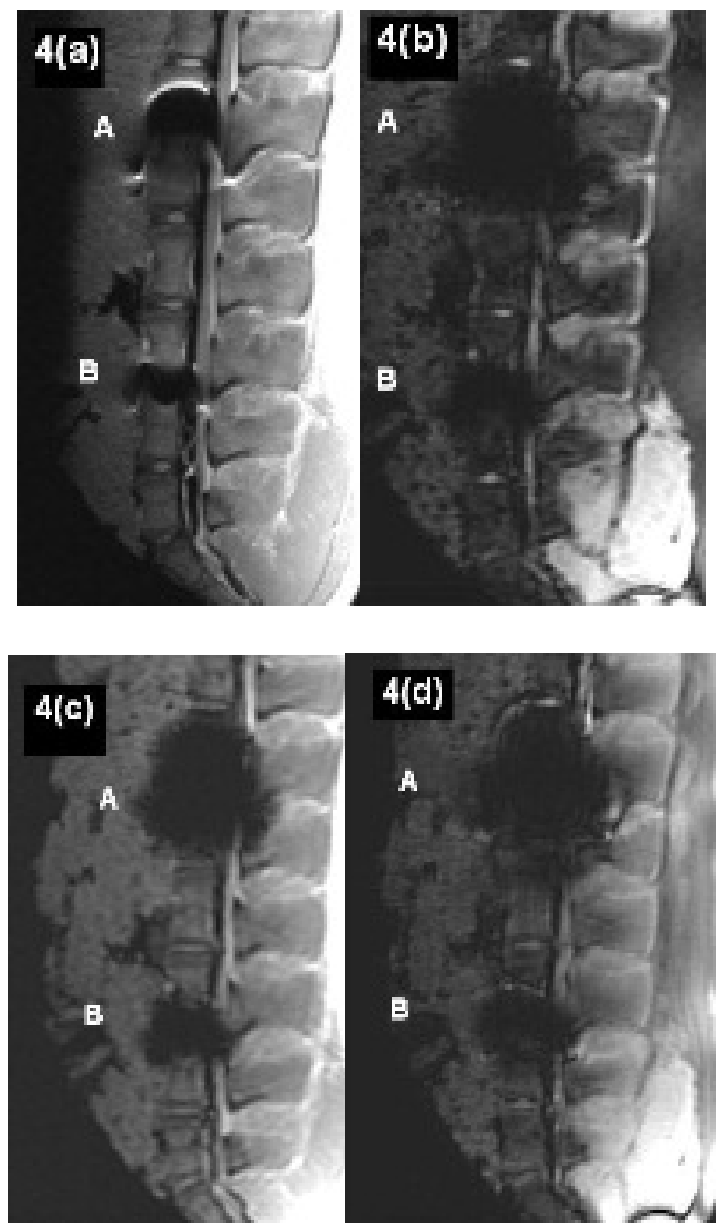

(a) T1 TSE (TR: 2260, TE: 14); (b) T2 DESS 3D (TR: 23, TE: 6); (c) T1 FLASH 3D FS (TR: 60, TE: 11); (d) TOF FISP 3D (TR: 36, TE: 4).

Figure 4.(a)-(d) Artifact range considering different MRIsequences. A: Cobalt-chromium-intervertebral disc dowel; B: IBS-titanium-spacer.

\subsection{Intervertebral Body Spacer (IBS LIBERTY)}

On account to T1 TSE, SE and T2 3D Sequences (Table 2) the IBS spacer could achieve a maximum score of 22 points respectively. With regard to the T1 SE and TSE sequences the susceptilibity artifact border could be clearly differentiated opposite the spinal surrounding. The regions of interest could be mainly represented to more than $50 \%$. On the other hand only a limited evaluation of the implant-bone contact area was possible considering the artifact extension. With regard to a differentiation of the spinal canal opposite the implant appropriate statements could be done. The achieved MRI image quality does not allow any concrete statements about the real implant position. The lowest MRI imaging quality with a maximum score of 15 points could be demonstrated considering T1 FLASH 3D FS sequence (Figure 4(d-B)). The existing susceptilibity artifacts guarantee an unsufficiently evaluation of the implant surrounding only.

\subsection{Summarizing Evaluation}

Independent on specific implant characteristics the best MRI imaging results for both metallic intervertebral disc spacers could be achieved considering fast spin echo sequences. A comparison of both metallic intervertebral disc spacers showed in all examined sequences clearly advantages in favour of the IBS titanium spacer. With regard to the favored fast spin echo sequences (T1 TSE) the IBS titanium pacer reached a total score of 15 points towards 12 points with the cobalt chromium intervertebral disc dowel.

\section{Discussion}

With regard to the MRI diagnostic of spinal changes different imaging sequences are available. In this context as a problem of special importance postoperative changes are to be considered after instrumented spine fusions. According to implant related artifacts an MRI imaging evaluation can be difficult. On account of documented disadvantages of bone grafts different intervertebral spacers are obtainable to supplement anterior spinal fusion [2-4]. In this context titanium alloys as well cobalt chromium alloys are of special interest. In contrast to bone grafting intervertebral disc spacer guarantee immediate load transmission with direct primary stability. Dependent on the amount of susceptibility artifacts an MRI based evaluation of the respective implant situation can be executed. A local magnetic field gradient results in the area between two different structures with varying susceptibility. In these sections the respective spins gyrate with different frequencies with the consequence of imaging distortions [3-7]. According to this a great susceptibility difference resulted in a limited imaging evaluation.

Regarding to material related implant charcteristics the following aspects are of special meaning in MRI imaging:

1) Evaluation of implant form and position;

2) Implant differentiation opposite anatomically neighbouring structures.

The MRI imaging behavior of metallic spinal implants is already documented in the literature [8-14]. However, the separate studies demonstrated partially great differences considering their purpose. Main aspect of most studies was the appreciation of the MRI-sequence related artifact size. Evaluation of intervertebral disc spacers considering clinical relevant aspects including possible implant malpositions were not examined so far. With regard to our MRI sequence record with a defined score we described the artifact range for every implant per MRI sequence. The score results of the titanium spacer 
as well as the cobalt chromium disc dowel are to be understood on the basis of increased susceptibility artifacts. Following the study results of Rudisch et al. [15] as well as Thomsen et al. [16] a lower artifact range could be noticed for titanium spacer in contrast to the cobalt chrome disc dowel. The best MRI imaging results of both metallic spacer could be achieved by using T1 TSE sequences. Additionally comparable results could be reproduced for the titanium spacer considering T1 SE and T2 CISS 3D sequences. Other MRI sequences according to the examination record demonstrated no further advantages. Studies with regard to metallic artifacts in the anterior spine were performed by Vaccaro et al. [17] and Wang et al. [13] so far. Vaccaro et al. [17] examined in a human carcass study the MRI artifact rates of different metal particles which were brought in defined intervertebrale drillings and subsequently parafin beded. On account of a lower particle density in contrast to clinical used metal implants Vacarro could not diagnosed any significant artifacts on account to T1 or T2 SE sequences. With regard to the metallic artifacts appeared in gradient echo-sequences a connection could be proved between the artifact size and the nickel content of the examined alloys. In this connection, an increasing nickel content effects a reduced susceptibility artifact expression.

Wang et al. [14] described in another carcass artifact study the MRI behavior of an intervertebral disc spacer made of titanium and tantalum. According to a correct implant position by using T1 SE sequences implant related artifacts of the titanium spacer could be limited to the direct implant surrounding. The anatomically representation of neighbouring structures under special consideration of the spinal canal could be achieved without any problems. With regard to equally MRI sequences the diagnosed metallic artifacts of the titanium spacer as well as the cobalt-chromium disc dowel in our study must be discussed controversially. Considering both metallic spacers by using spin echo-as well as fast spin echo sequences neither an evaluation of the implant form nor the implant position could be carried out certainly. Regarding to a phantom study of Rudisch et al. a relation between the range of metallic artifacts and implant related characteristics like material, form and position could be demonstrated beneath the selected MRI sequence. In this connection already published results of similar artifact studies seem to be dependent on the respective study concept. In spite of the use of comparable MRI-sequences considering the MRI imaging of metallic spine implants a variability of the amount of susceptibility artifacts has to be included.

\section{Conclusion}

According to anterior spine fusion available metallic intervertebral disc spacers made of titanium or cobalt- chrome alloys are of special interest. In spite of the use of favored fast spin echo MRI sequences a variability of the amount of susceptibility artifacts has to be included considering the MRI imaging of metallic spine implants. Taking into account already documented studies beneath the chosen MRI sequence the artifact size seems to be affected by the implant shape as well as the implant material. Regarding our study results the easy use of a new developed artefact score demonstrated a useful help to compare implant related MRI artifact properties independent from the actual implant related total artifact volume.

\section{REFERENCES}

[1] T. Herold, W. C. Caro, G. Heers, L. Perlick, J. Grifka, S. Feuerbach, W. Nitz and M. Lenhart, "Influence of Sequence Type on the Extent of the Susceptility Artifact in MRI-A Shoulder Specimen Study after Suture Anchor Repair,” Rofo, Vol. 176, No. 9, 2004, pp. 1296-1301. doi:10.1055/s-2004-813404

[2] J. W. Brantigan and A. D. Steffee, "A Carbon Fiber Implant to Aid Interbody Lumbar Fusion. Two-Year Clinical Results in the First 26 Patients,” Spine, Vol. 18, No. 14, 1993, pp. 2106-2107. doi:10.1097/00007632-199310001-00030

[3] J. A. Goulet, L. E. Senunas, G. L. DeSilva and M. L. Greenfield, "Autogenous Iliac Crest Bone Graft: Complications and Functional Assessment," Clinical Orthopaedics \& Related Research, Vol. 339, No. 339, 1997, pp. 76-81.

[4] B. N. Summers and S. M. Eisenstein, "Donor Site Pain from the Ilium. A Complication of Lumbar Spine Fusion," The Journal of Bone \& Joint Surgery, Vol. 71, No. 4, 1989, pp. 677-680.

[5] C. Fellner, M. Behr, F. Fellner, P. Held, G. Handel and S. Feuerbach, "Artifacts in MR Imaging of the Temporomandibular Joint Caused by Dental Alloys: A Phantom Model Study at T1.5,” Rofo, Vol. 166, No. 5, 1997, pp. 421-428. doi:10.1055/s-2007-1015452

[6] S. Fritzsche, R. Thull and A. Haase, "Reduction of Artifacts in Magnetic Resonance Images by Using Optimized Materials for Diagnostic Devices and Implants,” Biomed Tech (Berl), Vol. 3, No. 39, 1994, pp. 42-46.

[7] J. F. Schenck, “The Role of Magnetic Susceptibility in Magnetic Resonance Imaging: MRI Magnetic Compatibility of the First and Second Kinds," Medical Physics, Vol. 23, No. 6, 1996, pp. 815-850. doi:10.1118/1.597854

[8] C. B. Henk, W. Brodner, S. Grampp, M. Breitenseher, M. Thurnher, G. H. Mostbeck and H. Imhof, "The Postoperative Spine,” Top Magn Reson Imaging, Vol. 10, No. 4, 1999, pp. 247-64.

doi:10.1097/00002142-199908000-00006

[9] A. S. Malik, O. Boyko, N. Atkar and W. F. Young, “A Comparative Study of MR Imaging Profile of Titanium Pedicle Screws,” Acta Radiologica, Vol. 42, No. 3, 2001, pp. 291-293. doi:10.1080/028418501127346846 
[10] O. Ortiz, T. G. Pait, P. McAllister and K. Sauter, "Postoperative Magnetic Resonance Imaging with Titanium Implants of the Thoracic and Lumbar Spine," Neurosurgery, Vol. 38, No. 4, 1996, pp. 741-745.

[11] C. A. Petersilge, J. S. Lewin, J. L. Duerk, J. U. Yoo and A. J. Ghaneyem, "Optimizing Imaging Parameters for MR Evaluation of the Spine with Titanium Pedicle Screws," American Journal of Roentgenology, Vol. 166, No. 5, 1996, pp. 1213-1218.

[12] R. Rupp, N. A. Ebraheim, E. R. Savolaine and W. T. Jackson, "Magnetic Resonance Imaging Evaluation of the Spine with Metal Implants: General Safety and Superior Imaging with Titanium,” Spine, Vol. 18, No. 3, 1993, pp. 379-385.

[13] J. C. Wang, H. S. Sandhu, M. D. Yu, J. T. Minchew and R. B. Delamarter, "MR Parameters for Imaging Titanium Spinal Instrumentation,” Journal of Spinal Disorders \& Techniques, Vol. 10, No. 1, 1997, pp. 27-32.

[14] J. C. Wang, W. D. Yu , H. S. Sandhu, V. Tam and R. B. Delamarter, “A Comparison of Magnetic Resonance and
Computed Tomographic Image Quality after the Implantation of Tantalum and Titanium Spinal Instrumentation," Spine, Vol. 23, No. 15, 1998, pp. 1684-1688. doi:10.1097/00007632-199808010-00014

[15] A. Rudisch, C. Kremser, S. Peer, A. Kathrein, W. Judmaier and H. Daniaux, "Metallic Artifacts in Magnetic Resonace Imaging of Patients with Spinal Fusion: A Comparison of Implant Materials and Implant Sequences," Spine, Vol. 23, No. 6, 1998, pp. 692-699. doi:10.1097/00007632-199803150-00009

[16] M. Thomsen, U. Schneider, S. J. Breusch, J. Hansmann and M. Freund, "Artifacts and Ferromagnetism Dependent on Different Metal Alloys in Magnetic Resonance Imaging. An Experimental Study,” Der Orthopäde, Vol. 30, No. 8, 2001, pp. 540-544. doi:10.1007/s001320170063

[17] A. R. Vaccaro, R. M. Chesnut, G. Scuderi, J. F. Healy, J. B. Massie and S. R. Garfin, "Metallic Spinal Artifacts in Magnetic Resonance Imaging,” Spine, Vol. 11, No. 19, 1994, pp. 1237-1242. 